Hsp70, a protein upregulated during cellular stress, is a molecular chaperone that facilitates protein folding and antigen presentation within cells ${ }^{6}$. In vitro findings show that when released by necrotic cells, Hsp70 has a quite different role. It acts like a danger signal by enhancing maturation of dendritic cells (DCs), the most potent type of $\mathrm{APC}^{7}$. These studies show that Hsp70 can be a trigger for immune activation.

Millar et al. asked the next logical question: does Hsp70 also ring an alarm for the immune system in vivo? To do this, they used engineered mice expressing gp in the pancreas. gp is a viral nuclear protein from lymphocytic choriomeningitis virus. The mice also expressed a T-cell receptor specific for gp33, a region of gp. The authors found that these mice remained healthy even when injected with gp33 peptide. However, when the peptide was coinjected with Hsp70, the mice rapidly developed autoimmune diabetes, associated with inflammation and infiltration of T cells into the pancreatic islets.

Unlike LPS, Hsp70 did not upregulate costimulatory molecules on DCs. Nevertheless, Hsp70 helped APCs become better stimulators for T cells, although exactly how is unknown.

Although LPS coinjected with gp33 was also able to induce diabetes, the authors provide evidence that LPS and Hsp70 act through different routes. Diabetes mediated by gp33 and Hsp70 required the $\mathrm{APC}$ costimulatory ligand $\mathrm{CD} 40$, whereas diabetes induced by LPS and Hsp70 did not. Specifically, in the absence of CD40, Hsp70 was unable to upregulate production of the inflammatory cytokine IL-12 by DCs. In contrast, LPS-stimulated DCs seemed to release IL-12 in a manner independent of CD40.

The work by Millar et al. elegantly connects two autoimmunity paradigms for the first time in vivo. First, it is now clear that endogenous signals, which are known products of necrotic cells, promote autoimmune responses. Second, it is the response of APCs to these endogenous signals that triggers autoimmunity.

The study also leaves open some important questions. For example, there is no clear explanation for the finding that Hsp70-stimulated DCs require CD40 for IL-12 synthesis, whereas LPS-stimulated DCs do not. Although one report shows Hsp70 binding to CD40 (ref. 8), other studies show that TLRs mediate APC responses to Hsp70 (ref. 9). Moreover, APC-mediated IL-12 production has been shown to occur through the same toll-like receptor, TLR4, in response to both LPS and Hsp70 (ref. 9). Thus, the story may be more complicated than previously appreciated. The next step, therefore, may be to see whether Hsp70-mediated autoimmune responses occur in the transgenic mouse model in a TLR4-deficient background.

The initial inflammatory conditions that produce the endogenous danger signals leading to autoimmunity are also unclear. Normal cell death occurs by apoptosis, which inhibits DC maturation ${ }^{10}$ (Fig. 1). The release of endogenous inflammatory signals (like Hsp70) requires necrotic cell death, such as from infection or injury. One notion is that exogenous PAMPs displayed by infectious agents create the initial conditions for autoimmunity, and this in turn leads to necrotic cell death, which perpetuates the autoimmune response. Selected clinical observations fall in line with this notion, as does the finding that the transgenic mice used by Millar et al. can also acquire diabetes through infection with lymphocytic choriomeningitis virus ${ }^{11}$. Whether or not endogenously generated danger signals contribute to perpetuation of the inflammatory process in autoimmunity is not known, although the Millar study indicates that this is a real possibility.

Despite these limitations in our knowledge, one of the most exciting findings of the new work is that CD40 is necessary for autoimmunity induced by Hsp70, but not by LPS. This finding suggests that APCs can discriminate between exogenous and endogenous maturation agents via CD40 signaling. If future studies support this observation, CD40 and its signaling pathway could become targets for the therapeutic intervention of autoimmune disease, perhaps enabling selective sparing of host antipathogen responses.

1. Millar, D. et al. Nat. Med. 9, 1469-1476 (2003)

2. Liblau, R.S., Wong, F.S., Mars, L.T. \& Santamaria, P. Immunity 17, 1-6 (2002).

3. Medzhitov, R. \& Janeway, C.A., Jr. Science 296, 298-300 (2002).

4. Beg, A.A. Trends Immunol. 23, 509-512 (2002).

5. Gallucci, S. \& Matzinger, P. Curr. Opin. Immunol. 13, 114-119 (2001).

6. Srivastava, P.K., Menoret, A., Basu, S., Binder, R.J. \& McQuade, K.L. Immunity 8, 657-665 (1998).

7. Basu, S., Binder, R.J., Suto, R., Anderson, K.M. \& Srivastava, P.K. Int. Immunol. 12, 1539-1546 (2000).

8. Becker, T., Hartl, F.U. \& Wieland, F. J. Cell Biol. 158, 1277-1285 (2002).

9. Vabulas, R.M. et al. J. Biol. Chem. 277, 15107-15112 (2002).

10. Stuart, L.M. et al. J. Immunol. 168, 1627-1635 (2002).

11. Ehl, S. et al. J. Exp. Med. 187, 763-774 (1998).

\title{
Push me, pull you
}

In the breast, cells are faced with the existential question common to many cells: differentiate or proliferate? In the 10 November Journal of Cell Biology, Michele Wozniak et al. consider this question in an in vitro system of epithelial breast differentiation. They examine how the flexibility of the extracellular matrix influences this critical decision.

It has been known for years that breast epithelial cells differentiate into tubules (at right) if the cells are cultured in a three-dimensional collagen matrix floating in medium. But if the matrix is made more rigid by attachment to a surface, or more dense by increasing the collagen concentration, proliferation is favored. How do breast cells sense the flexibility of their surroundings? The investigators found that cells use contractile forces, generated through the small GTPase Rho, to sense structural stretch in the extracellular matrix. In a flexible matrix, cell contraction downregulates the small GTPase Rho and the focal adhesion kinase (FAK). In a rigid matrix, Rho is upregulated and FAK is enlisted to help clamp down the cells at matrix adhesion sites (inset; FAK in red, nuclei in blue). In addition to regulating cell stickiness, FAK also links to pathways that regulate proliferation and migration.

In the breast, epithelial cells must contend with numerous inputs. But in some women, cells face particularly dense breast tissue and high deposition of collagen. These women have a four- to six-fold increased risk of developing breast cancer.

Charlotte Schubert 